01.1;01.4;05.2;09.1;15.2

\title{
Сдвиг инфракрасных спектров поглощения и излучения ионов переходных металлов в твердых растворах полупроводниковых соединений
}

\author{
(C) C.B. Найденов \\ Институт монокристаллов НАН Украины, Харьков, Украина \\ E-mail: sergei.naydenov@gmail.com
}

Поступило в Редакцию 18 февраля 2021 г.

В окончательной редакции 21 марта 2021 г.

Принято к публикации 26 марта 2021 г.

Предложена универсальная теоретическая модель, объясняющая эффект сдвига инфракрасных полос поглощения и излучения ионов переходных металлов в твердых растворах полупроводниковых соединений. На примере кристаллов тройных соединений $\mathrm{Zn}_{1-x} \mathrm{Mg}_{x} \mathrm{Se}: \mathrm{Cr}^{2+}$ и $\mathrm{Cd}_{1-x} \mathrm{Mn}_{x} \mathrm{Te}_{\mathrm{Fe}} \mathrm{Fe}^{2+}$ дана оценка параметров длинноволнового сдвига полос люминесценции с ростом концентрации твердого раствора. Рассмотрено обобщение эффекта на случай многокомпонентных твердых растворов.

Ключевые слова: твердые растворы полупроводниковых соединений, монокристаллы $\mathrm{A}^{\mathrm{II}} \mathrm{B}^{\mathrm{VI}}: \mathrm{TM}^{2+}$, лазерные приложения для среднего ИК-диапазона, люминесценция ионов переходных металлов, внутрицентровый переход.

DOI: 10.21883/PJTF.2021.12.51068.18742

Кристаллические материалы полупроводниковых соединений группы $\mathrm{A}^{\mathrm{II}} \mathrm{B}^{\mathrm{VI}}: \mathrm{TM}^{2+}$, допированные ионами переходных металлов, относятся к перспективным материалам для лазерных сред ближнего и среднего инфракрасного (ИК) диапазона [1,2]. В различных приложениях требуется разработка ИК-лазерных сред с широкой перестраиваемой полосой генерации, смещенной в сторону больших длин волн (вплоть до 5-6 $\mathrm{m}$ ). Новые возможности по сравнению с бинарными соединениями предоставляют твердые растворы сложных халькогенидов, в которых основной катион кристаллической решетки замещается более легким атомом. В частности, вызывают интерес кристаллы $\mathrm{Zn}_{1-x} \mathrm{Mg}_{x} \mathrm{Se}: \mathrm{Cr}^{2+}$ [3], $\mathrm{Zn}_{1-x} \mathrm{Mn}_{x} \mathrm{~S}: \mathrm{Fe}^{2+}$ [4], $\mathrm{Cd}_{1-x} \mathrm{Mn}_{x} \mathrm{Te}: \mathrm{Cr}^{2+}$ [5], $\mathrm{Cd}_{1-x} \mathrm{Mn}_{x} \mathrm{Te}: \mathrm{Fe}^{2+}[6,7]$, на которых была получена лазерная генерация в среднем ИК-диапазоне. Отличительной чертой этих кристаллов является существенный сдвиг полос поглощения и люминесценции ионов переходного металла при изменении катионной концентрации твердого раствора ( $\propto 20-50 \mathrm{~nm}$ на каждые 10\% прироста концентрации $x$ ) и температуры (в меньшей степени). Благодаря этому открывается возможность контролируемого изменения их лазерных свойств и сдвига полосы лазерной генерации в сторону больших длин волн. Физическая природа этого эффекта не была выяснена в достаточной степени. Цель настоящей работы - построить теоретическую модель, объясняющую наблюдаемый эффект, и связать микроскопические параметры электронной энергетической структуры с экспериментальными данными.

Рассмотрим люминесценцию изовалентных примесей переходных металлов $\mathrm{TM}^{2+}(\mathrm{Cr}, \mathrm{Fe}, \mathrm{Co}, \mathrm{Ni}$ и др.), которыми допируются кристаллы соединений группы $\mathrm{A}^{\mathrm{II}} \mathrm{B}^{\mathrm{VI}}: \mathrm{TM}^{2+}$. Поскольку различие между ионными радиусами и электроотрицательностью $\mathrm{TM}^{2+}$-ионов и катионов кристаллической решетки ( $\mathrm{Zn}, \mathrm{Cd}, \mathrm{Hg}$ и др.) обычно не превосходит $10-15 \%$ и $0.4-0.6$ единиц Поллинга соответственно, ион переходного металла, согласно правилу Гольдшмидта, изоморфно замещает катион кристаллической решетки. В результате замещения „центральный ион“ оказывается в октаэдрическом или тетраэдрическом (в зависимости от типа решетки) окружении соседних анионов халькогена $(\mathrm{S}, \mathrm{Se}, \mathrm{Te})$. При этом уровни примесного иона оказываются расположенными глубоко в запрещенной зоне полупроводника $[8,9]$. Люминесценция $\mathrm{TM}^{2+}$-ионов в области ИК-диапазона имеет внутрицентровой характер. Процессы перезарядки $\mathrm{TM}^{2+}$-ионов при облучении коротковолновым излучением видимого и ультрафиолетового диапазона или в результате ударной ионизации свободными электронами (в сильном электрическом поле порядка нескольких киловольт) и связанную с ними рекомбинационную (коротковолновую) люминесценцию здесь не рассматриваем.

Кристаллическое поле лигандов приводит к расщеплению энергетических уровней центрального иона (см., например, [10]). Для изолированного тетраэдрического комплекса энергия расщепления $\Delta E^{*}$ выражается формулой Бете (в системе единиц СИ)

$$
\Delta E^{*}=\frac{20}{27} \frac{e^{2} Z^{2}}{4 \pi \epsilon_{0} \epsilon} \frac{\left\langle r^{4}\right\rangle}{d^{* 5}},
$$

где $\epsilon_{0}=8.85 \cdot 10^{-12} \mathrm{~F} / \mathrm{m}$ - диэлектрическая постоянная вакуума, $\epsilon$ - диэлектрическая постоянная среды, $e=1.6 \cdot 10^{-19} \mathrm{C}$ - заряд электрона, $Z$ - заряд лиганда 
Таблица 1. Параметры [2] бинарных кристаллов $\mathrm{A}^{\mathrm{II}} \mathrm{B}^{\mathrm{VI}}: \mathrm{TM}^{2+}$ и рассчитанная для них энергия расщепления $\Delta E^{*}$ внутрицентрового перехода $\mathrm{TM}^{2+}$-иона

\begin{tabular}{|c|c|c|c|c|c|c|c|c|c|}
\hline \multirow{2}{*}{ Параметр } & \multicolumn{5}{|c|}{ Допированные ионами $\mathrm{Cr}^{2+}$} & \multicolumn{4}{|c|}{ Допированные ионами $\mathrm{Fe}^{2+}$} \\
\hline & $\mathrm{ZnS}$ & $\mathrm{ZnSe}$ & $\mathrm{ZnTe}$ & $\mathrm{CdS}$ & $\mathrm{CdSe}$ & $\mathrm{ZnS}$ & $\mathrm{ZnSe}$ & $\mathrm{CdSe}$ & CdTe \\
\hline $\begin{array}{c}\text { Тип кристаллической } \\
\text { решетки }\end{array}$ & ZB & ZB & ZB & W & W & ZB & ZB & W & $\mathrm{ZB}$ \\
\hline Параметр решетки, А & 5.41 & 5.67 & 6.10 & $\begin{array}{l}c=6.75 \\
a=4.14\end{array}$ & $\begin{array}{l}c=7.02 \\
a=4.30\end{array}$ & 5.41 & 5.67 & $\begin{array}{l}c=7.02 \\
a=4.30\end{array}$ & 6.48 \\
\hline $\begin{array}{c}\text { Ширина запрещенной } \\
\text { щели, } \mathrm{eV}\end{array}$ & 3.7 & 2.7 & 2.3 & 2.5 & 1.7 & 3.7 & 2.7 & 1.7 & 1.5 \\
\hline Пик поглощения $\lambda_{a b}, \mu \mathrm{m}$ & 1.69 & 1.77 & 1.79 & 1.85 & 1.92 & 2.8 & 3.1 & 3.5 & 3.65 \\
\hline Пик излучения $\lambda_{e m}, \mu \mathrm{m}$ & 2.35 & 2.45 & 2.4 & 2.6 & 2.75 & 3.94 & 4.35 & 4.81 & 5.94 \\
\hline $\begin{array}{c}\text { Энергия расщепления } \Delta E^{*}, \\
\mathrm{eV}\end{array}$ & 0.464 & 0.442 & 0.438 & 0.393 & 0.371 & 0.286 & 0.253 & 0.216 & 0.192 \\
\hline
\end{tabular}

(равный заряду иона) в единицах заряда электрона, $\left\langle r^{4}\right\rangle$ - матричный элемент от четвертой степени $r^{4}$ расстояния валентного $d$-электрона от ядра иона, вычисленный по волновой функции многочастичной системы (центрального иона со всеми его электронами), $d^{*}-$ расстояние между центральным ионом и лигандами, т.е. длина связей в кристаллической решетке. Для кубической решетки бинарного соединения (типа сфалерит) $d^{*}=(\sqrt{3} / 4) a$. В общем случае можно считать, что параметр $d^{*} \propto d$ пропорционален периоду кристаллической решетки.

Для бинарных кристаллов $\mathrm{A}^{\mathrm{II}} \mathrm{B}^{\mathrm{VI}}: \mathrm{TM}^{2+}$ формулу (1) можно считать хорошим приближением для оценки энергетической щели между электронными уровнями основного и возбужденного состояний $\mathrm{TM}^{2+}$-иона. Спин-орбитальное взаимодействие при искажении решетки и взаимодействие решетки с электронной подсистемой (вибронные возбуждения) в силу эффекта Яна-Теллера приводят к дополнительному расщеплению уровней $d$-электронов $\mathrm{TM}^{2+}$-иона, которые размываются в достаточно широкие подзоны. Обычно величина $\Delta E^{*} \propto 10^{3} \mathrm{~cm}^{-1}$, тогда как для вибронных уровней $\Delta E_{v} \propto 1-10 \mathrm{~cm}^{-1}$.

Внутрицентровый переход $\mathrm{TM}^{2+}$-иона при поглощении и излучении в ИК-диапазоне происходит между подзонами основного и возбужденного состояний иона, разделенными энергетической щелью $\Delta E^{*}$. Из формулы (1) следует, что с ростом параметра $d^{*}$, а значит, c ростом параметра решетки бинарного соединения $\mathrm{A}^{\mathrm{II}} \mathrm{B}^{\mathrm{VI}}: \mathrm{TM}^{2+}$ величина $\Delta E^{*}$ уменьшается. Поэтому максимумы полос поглощения и излучения должны сдвигаться в длинноволновую область. В табл. 1 приведены экспериментальные и расчетные данные (детали расчета опускаем) для серии бинарных лазерных кристаллов $\mathrm{ZnS}, \mathrm{ZnSe}, \mathrm{ZnTe}, \mathrm{CdS}, \mathrm{CdSe}, \mathrm{CdTe}$, допированных ионами хрома и железа. При переходе от более легких к более тяжелым катионам и/или анионам ковалентная связь в решетке ослабляется (заменяется на ионную), параметр решетки возрастает (ширина запрещенной зоны при этом падает, так как ослабляется ковалентная связь); ширина энергетической щели $\Delta E^{*}$ внутрицентрового перехода также падает: ИК-полосы поглощения и излучения согласованно сдвигаются в сторону больших длин волн. Несмотря на простоту и очевидность последнего вывода, цитируемого в большом числе работ по лазерным кристаллам $\mathrm{A}^{\mathrm{II}} \mathrm{B}^{\mathrm{VI}}: \mathrm{TM}^{2+}$, данная закономерность напрочь отсутствует в кристаллах твердых растворов тройных соединений. Например, для $\mathrm{Zn}_{1-x} \mathrm{Mg}_{x} \mathrm{Se}: \mathrm{Cr}^{2+}$ с увеличением концентрации магния постоянная решетки растет, а для $\mathrm{Cd}_{1-x} \mathrm{Mn}_{x} \mathrm{Te}: \mathrm{Fe}^{2+}$ с увеличением концентрации марганца, наоборот, падает. Вместе с тем для обоих кристаллов по-прежнему наблюдается длинноволновый сдвиг ИК-полос поглощения и излучения [3-7].

В твердом растворе замещения типа $A_{1-x} B_{x} C: \mathrm{TM}^{2+}$ (здесь $A$ и $B$ - катионы основной решетки и разбавителя, $C-$ халькогена, $x-$ концентрация твердого раствора) примесный ион может замещать не один, а любой из двух сортов катионов. Энергия расщепления $\Delta E^{*}$ уровней $\mathrm{TM}^{2+}$-иона зависит от этого локального окружения. Поэтому в данном случае она не может выражаться формулой (1). Для того чтобы связать ее с параметрами полупроводникового материала, воспользуемся тем, что физическая величина при переходе от простых соединений к их твердому раствору должна изменяться (в первом приближении) пропорционально их концентрации в твердом растворе. В частном случае этот принцип, примененный к параметру решетки $d=d(x)$, соответствует известному закону Вегарда $d(x)=d_{1}+\left(d_{2}-d_{1}\right) x$, который неплохо выполняется для исследуемых материалов.

В применении к энергетической щели $\Delta E^{*}$ между состояниями примесного $\mathrm{TM}^{2+}$-иона указанный принцип аддитивности означает

$$
\Delta E_{A B C: \mathrm{TM}}^{*}(x)=(1-x) \Delta E_{A C: \mathrm{TM}}^{*}+x \Delta E_{B C: \mathrm{TM}}^{*} .
$$


Таблица 2. Параметры бинарных компонентов $(i=1,2)$ твердого раствора $A B C: \mathrm{TM}^{2+}$ (диэлектрическая проницаемость $\epsilon$, ионный радиус $r$ катиона, параметр решетки $d$ ) и параметры длинноволнового сдвига $\delta_{12}$ и $\kappa$ для внутрицентрового перехода (в качестве радиуса $r_{I}$ валентного $d$-электрона ТМ-иона для оценок выбран радиус примесного атома)

\begin{tabular}{c|c|c|c|c|c|c|c|c|c}
\hline Кристалл & $\epsilon_{1}$ & $\epsilon_{2}$ & $r_{1}, \AA$ & $r_{2}, \AA$ & $d_{1}, \AA$ & $d_{2}, \AA$ & $r_{I}, \AA$ & $\delta_{12}, \mathrm{eV}$ & $\kappa$ \\
\hline $\mathrm{ZnMgSe}: \mathrm{Cr}^{2+}$ & 7.32 & 4.66 & 0.74 & 0.71 & 5.67 & 6.88 & 1.27 & $6.84 \cdot 10^{-2}$ & $1.55 \cdot 10^{-1}$ \\
$\mathrm{CdMnTe}: \mathrm{Fe}^{2+}$ & 12.82 & 19.30 & 0.92 & 0.80 & 6.48 & 6.33 & 1.26 & $1.22 \cdot 10^{-2}$ & $6.36 \cdot 10^{-2}$
\end{tabular}

В рамках этого подхода можно также учесть влияние температуры, добавив к выражению (2) слагаемое $\propto T$ (здесь не рассматриваем). Переобозначив величины, запишем (2) в виде

$$
\varepsilon(x)=\varepsilon_{1}^{*}-\delta_{12} x, \quad \delta_{12}=\left(\varepsilon_{1}^{*}-\varepsilon_{2}^{*}\right),
$$

Энергия $\varepsilon(x)$ соответствует энергии поглощенного или излученного фотона при внутрицентровом переходе $\mathrm{TM}^{2+}$-иона в матрице твердого раствора с концентрацией $x$. Изменение длины волны $\Delta \lambda$ связано с изменением энергии фотона $\Delta \varepsilon$ соотношением

$$
\Delta \lambda / \lambda=-\Delta \varepsilon / \varepsilon
$$

При небольшом приращении величин $\Delta \lambda \ll \lambda$ или $\Delta \varepsilon \ll \varepsilon$ из уравнений (3), (4) следует

$$
\Delta \lambda \approx \lambda \kappa \Delta x, \quad \kappa=\delta_{12} / \varepsilon_{1}^{*} \ll 1
$$

- закон линейного сдвига максимума полосы поглощения или излучения при изменении концентрации $\Delta x$ твердого раствора. Значения $\lambda$ соответствуют максимумам в спектрах поглощения $\lambda_{a b}$ или излучения

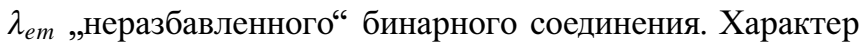
сдвига определяется знаком величины $\delta_{12}$. Длинноволновому спектральному сдвигу соответствует $\delta_{12}>0$, а коротковолновому $-\delta_{12}<0$. В общем случае значение коэффициента сдвига $\kappa=\kappa_{\alpha}$ зависит от структуры зонного энергетического спектра ТМ-иона в матрице твердого раствора. Для каждой выделенной $\alpha$-й полосы сложного спектра поглощения или излучения он может принимать различные (хотя, как правило, близкие) значения. Основной вклад вносит штарковское расщепление уровней, т. е. величина $\Delta E^{*}(x)$.

В табл. 2 приведена упрощенная оценка параметpa $\delta_{12}$ для тройных кристаллов $\mathrm{Zn}_{1-x} \mathrm{Mg}_{x} \mathrm{Se}_{\mathrm{C}} \mathrm{Cr}^{2+}$ и $\mathrm{Cd}_{1-x} \mathrm{Mn}_{x} \mathrm{Te}: \mathrm{Fe}^{2+}$. Для обоих соединений $\delta_{12}>0$, т.е. сдвиг полос является длинноволновым. Как видно из табл. 1, 2, по порядку величины $\varepsilon^{*} \propto 10^{-1} \mathrm{eV}$, $\delta \propto 10^{-2} \mathrm{eV}$ и $\kappa \propto 10^{-1}$.

Для ИК-диапазона $\lambda \propto 10^{3} \mathrm{~nm}$. Отсюда следует, что максимальный сдвиг полос при изменении концентрации $\Delta x \propto 1$ может достигать нескольких сотен нанометров, $\Delta \lambda \propto 10^{2} \mathrm{~nm}$. По порядку величины эта оценка справедлива и для других ИК-лазерных кристаллов на основе тройных халькогенидов. „Красный“ сдвиг максимумов полос люминесценции у кристаллов $\mathrm{Cd}_{1-x} \mathrm{Mn}_{x} \mathrm{Te}: \mathrm{Fe}^{2+}$ в несколько раз меньше, чем у кристаллов $\mathrm{Zn}_{1-x} \mathrm{Mg}_{x} \mathrm{Se}: \mathrm{Cr}^{2+}$, так как у них сильно отличается параметр $\delta_{12}$ и коэффициент сдвига $\kappa$ уменьшается в 2.4 раза (в рамках теоретической оценки), что соответствует наблюдаемому в эксперименте уменьшению величины сдвига в 2-2.5 раза.

Линейная зависимость (5) и оценки подтверждаются в эксперименте (см., например, [3-7]). В этих работах установлен длинноволновый сдвиг полос поглощения и люминесценции на $\propto 50 \mathrm{~nm}$ (кристаллы $\mathrm{Zn}_{1-x} \mathrm{Mg}_{x} \mathrm{Se}: \mathrm{Cr}^{2+}$ ) или на $\propto 20-25 \mathrm{~nm}$ (кристаллы $\left.\mathrm{Cd}_{1-x} \mathrm{Mn}_{x} \mathrm{Te}: \mathrm{Fe}^{2+}\right)$ при изменении концентрации твердого раствора (магния или марганца соответственно) на каждые $10 \%$. Отсюда следует, что коэффициент пропорциональности для линейного сдвига полос поглощения и излучения соответственно принимает значения $\kappa_{a b} \approx 2.82 \cdot 10^{-1}, \kappa_{e m} \approx 2.04 \cdot 10^{-1}$ для $\mathrm{Zn}_{1-x} \mathrm{Mg}_{x} \mathrm{Se}: \mathrm{Cr}^{2+}$ (максимумы поглощения и излучения при 1770 и $2450 \mathrm{~nm})$ и $\kappa_{a b} \approx 6.85 \cdot 10^{-2}$, $\kappa_{e m} \approx 4.21 \cdot 10^{-2}$ для $\mathrm{Cd}_{1-x} \mathrm{Mn}_{x} \mathrm{Te}: \mathrm{Fe}^{2+}$ (максимумы поглощения и излучения при 3650 и $5940 \mathrm{~nm})$. По порядку величины эти значения соответствуют расчетным данным в табл. 2. Различия связаны с грубым характером оценки параметров кристаллического поля, не учитывающей помимо штарковского дальнейшее расщепление вырожденных состояний в основной и возбужденной зоне энергий ТМ-иона. Последнее приводит к размытию (уширению) спектра уровней ТМ-иона и расщеплению спектров поглощения и излучения на несколько перекрывающихся полос. Параметры указанного изменения энергетической структуры зависят от концентрации твердого раствора. Однако для каждой из полос линейная зависимость вида (5), каждая со своим параметром сдвига $\kappa_{\alpha}$, сохраняется.

Отметим, что присутствующие в твердых растворах соединений $\mathrm{A}^{\mathrm{II}} \mathrm{B}^{\mathrm{VI}}: \mathrm{TM}^{2+}$ примеси и дефекты могут влиять на свойства зонной структуры и люминесценцию, особенно в коротковолновой области. Условия применения лазерных кристаллов в ИК-области требуют высокой степени очистки этих кристаллов от посторонних примесей, которые могут приводить к росту концентрации свободных носителей заряда и ухудшению оптической ИК-прозрачности кристаллов из-за рассеяния ИК-излучения на электронах. Для хороших лазерных 
кристаллов на основе твердых растворов $\mathrm{A}^{\mathrm{II}} \mathrm{B}^{\mathrm{VI}}$ концентрация вредных примесей обычно составляет менее $10^{-2}-10^{-5 \%}$ (степень очистки сырья от $3 \mathrm{~N}$ до $6 \mathrm{~N}$ ), что значительно меньше концентрации активных ТМионов, которая составляет величину порядка нескольких процентов в матрице. Поэтому влиянием посторонних примесей на рассмотренные эффекты можно пренебречь.

Обнаруженную закономерность можно распространить на многокомпонентные твердые растворы типа $A\left(x_{1}\right)\left\{B_{i}\left(x_{i}\right)\right\} C\left(y_{1}\right)\left\{D_{j}\left(y_{j}\right)\right\}: \mathrm{TM}^{2+}$, в которые кроме основного катиона с концентрацией $x_{1}$ и основного аниона с концентрацией $y_{1}$ входят еще $i=2, \ldots, N$ катионов с концентрацией $x_{i}$ и $j=2, \ldots, M$ анионов с концентрацией $y_{j}$. Например, к ним относятся кристаллы $\mathrm{Zn}_{1-x} \mathrm{Mg}_{x} \mathrm{Se}_{1-y} \mathrm{~S}_{y}: \mathrm{Cr}^{2+}$ или $\mathrm{Cd}_{1-x} \mathrm{Mn}_{x} \mathrm{Te}_{1-y} \mathrm{Se}_{y}: \mathrm{Fe}^{2+}$. Ожидаемый сдвиг энергетической щели для состояний $\mathrm{TM}^{2+}$-иона

$$
\begin{aligned}
\varepsilon\left(\left\{x_{i}\right\},\left\{y_{j}\right\}\right) & =\varepsilon_{11}-\sum_{i=2}^{N} \delta_{i} x_{i}-\sum_{j=2}^{M} \gamma_{j} y_{j} \\
& +\sum_{j=2}^{N} \sum_{j=2}^{M} \xi_{i j} x_{i} y_{j},
\end{aligned}
$$

где $\varepsilon_{11}$ - энергия расщепления $\Delta E^{*}$ для основного компонента $A C$, а также введены параметры

$$
\begin{gathered}
\delta_{i}=\delta_{i}\left(y_{1}\right)=\varepsilon_{11}-y_{1} \varepsilon_{i 1}, \gamma_{j}=\gamma_{j}\left(x_{1}\right)=\varepsilon_{11}-x_{1} \varepsilon_{1 j}, \\
\xi_{i j}=\varepsilon_{11}+\varepsilon_{i j} \geqslant 0,
\end{gathered}
$$

где $\varepsilon_{i 1}, \varepsilon_{1 j}, \varepsilon_{i j}$ - энергии $\Delta E^{*}$ для бинарных компонентов $B_{i} C, A D_{j}, B_{i} D_{j}$ соответственно. Концентрации компонентов твердого раствора удовлетворяют условию нормировки

$$
x_{1}=1-\sum_{i=2}^{N} x_{i}, \quad y_{1}=1-\sum_{j=2}^{M} y_{j} .
$$

В многокомпонентной системе с почти „равноправным“ парциальным составом $x_{i} \propto 1 / N$ и $y_{j} \propto 1 / M$. Поэтому при $N>2$ и $M>2$ нелинейным слагаемым $\propto x_{i} y_{j} \ll 1$ в уравнении (7) можно пренебречь. Ожидаемый „квазилинейный“ сдвиг полос поглощения и излучения

$$
\begin{gathered}
\Delta \lambda\left(\left\{x_{i}\right\},\left\{y_{j}\right\}\right) \approx \lambda\left[\sum_{i=2}^{N} \kappa_{i} \Delta x_{i}+\sum_{j=2}^{M} v_{j} \Delta y_{j}\right], \\
\kappa_{i}=\frac{\delta_{i}}{\varepsilon_{11}}, v_{j}=\frac{\gamma_{j}}{\varepsilon_{11}} .
\end{gathered}
$$

В зависимости от знака параметров $\delta_{i}$ и $\gamma_{j}$ возможно $(N+M-2)$ типов поведения ИК-спектров при изменении концентрации компонентов твердого раствора. В самом простом случае $N=2, M=1$ зависимость (9) переходит в закон (5) для тройного соединения. Максимальный сдвиг ИК-спектров соответствует соединению, для которого параметры „сдвига“ $\delta_{i}$ и $\gamma_{j}$, а значит, и параметры $\kappa_{i}$ и $v_{j}$ принимают максимальные значения

$$
\Delta \lambda\left(\left\{x_{i}\right\},\left\{y_{j}\right\}\right) \leqslant \lambda\left[\kappa_{\max } \Delta x_{\max }+v_{\max } \Delta y_{\max }\right] .
$$

Для тройных соединений величину спектрального сдвига также можно оценить на основе экспериментальных измерений максимумов полос поглощения $\lambda_{a b}$ и излучения $\lambda_{e m}$ на двух образцах кристаллов с разной концентрацией твердого раствора $x_{1} \neq x_{2}$ по формуле

$$
\Delta \lambda=\frac{\lambda_{2}-\lambda_{1}}{x_{2}-x_{1}} \Delta x
$$

причем одна из концентраций может соответствовать бинарному составу $\left(x_{1}=0\right)$. Для $(N+M)$-компонентной системы для этого потребуется $(N+M-2)$ измерений, чтобы восстановить значения всех параметров сдвига в законе (9), т.е. решить „обратную“ задачу.

Полученные закономерности позволяют предсказать ожидаемое смещение полос ИК-люминесценции твердых растворов халькогенидов без предварительного выращивания самих кристаллов. Это может оказаться полезным при разработке новых лазерных сред среднего ИК-диапазона.

\section{Конфликт интересов}

Автор заявляет, что у него нет конфликта интересов.

\section{Список литературы}

[1] I.T. Sorokina, in Solid-state mid-infrared laser sources, ed. by I.T. Sorokina, K.L. Vodopyanov. Topics in Applied Physics (Springer, Berlin-Heidelberg, 2003), vol. 89, p. 255-351. DOI: $10.1007 / 3-540-36491-9 \_7$

[2] S.B. Mirov, I.S. Moskalev, S. Vasilyev, V. Smolski, V.V. Fedorov, D. Martyshkin, J. Peppers, M. Mirov, A. Dergachev, V. Gapontsev, IEEE J. Sel. Top. Quantum Electron., 24 (5), 1601829 (2018). DOI: $10.1109 /$ JSTQE.2018.2808284

[3] M.E. Doroshenko, V.V. Osiko, H. Jelinkova, M. Jelinek, M. Nemec, J. Sulc, N.O. Kovalenko, A.S. Gerasimenko, V.M. Puzikov, Opt. Mater., 47, 185 (2015). DOI: $10.1016 /$ j.optmat.2015.05.015

[4] A.D. Martinez, D.V. Martyshkin, R.P. Camata, V.V. Fedorov, S.B. Mirov, Opt. Mater. Express, 5 (9), 2036 (2015). DOI: 10.1364/OME.5.002036

[5] U. Hommerich, X. Wu, V.R. Davis, S.B. Trivedi, K. Grasza, R.J. Chen, S. Kutcher, Opt. Lett., 22 (15) 1180 (1997). DOI: $10.1364 /$ OL.22.001180

[6] M.E. Doroshenko, V.V. Osiko, H. Jelinkova, M. Jelinek, J. Sulc, D. Vyhlidal, N.O. Kovalenko, I.S. Terzin, Opt. Mater. Express, 8 (7), 1708 (2018). DOI: 10.1364/OME.8.001708

[7] M.E. Doroshenko, H. Jelinkova, M. Jelinek, J. Sulc, D. Vyhlidal, N.O. Kovalenko, I.S. Terzin, Opt. Lett., 43 (20), 5058 (2018). DOI: 10.1364/OL.43.005058 
[8] A. Zunger, Solid state physics, ed. by H. Ehrenreich, D. Turnbull (Academic Press, 1986), vol. 39, p. 275. DOI: 10.1016/S0081-1947(08)60371-9

[9] T.P. Surkova, M. Godlewski, K. Swiatek, P. Kaczor, A. Polimeni, L. Eaves, W. Giriat, Physica B, 273-274, 848 (1999). DOI: 10.1016/S0921-4526(99)00519-0

[10] И.Б. Берсукер, Электронное строение и свойства координационных соединений. Введение в теорию, 3-е изд. (Химия, Л., 1986), с. 69, 269. 